\title{
Mutation spectrum and differential gene expression in cystic and solid vestibular schwannoma
}

\author{
Zhihua Zhang, PhD ${ }^{1,2}$, Zhaoyan Wang, $\mathrm{PhD}^{1,2}$, Lianhua Sun, MS 1,2 , Xiaohua Li, PhD ${ }^{1,2}$, \\ Qi Huang, BS ${ }^{1,2}$, Tao Yang, PhD ${ }^{1,2}$ and Hao $\mathrm{Wu}, \mathrm{PhD}, \mathrm{MD}^{1,2}$
}

Purpose: We sought to characterize the mutation spectrum of NF2 and the differential gene expression in cystic and solid vestibular schwannomas.

Methods: We collected tumor tissue and blood samples of 31 cystic vestibular schwannomas and 114 solid vestibular schwannomas. Mutation screening of NF2 was performed in both tumor and blood DNA samples of all patients. cDNA microarray was used to analyze the differential gene expression between 11 cystic vestibular schwannomas and 6 solid vestibular schwannomas. Expression levels of top candidate genes were verified by quantitative reverse transcription PCR.

Results: NF2 mutations were identified in $34.5 \%$ of sporadic vestibular schwannomas, with all mutations being exclusively somatic. No significant difference was found between the mutation detection rates of cystic vestibular schwannoma (35.5\%) and solid vestibular schwannoma (34.2\%). cDNA microarray analysis detected a total of 46 differentially expressed genes between the cystic vestibular schwannoma and solid vestibular schwannoma samples. The significantly decreased expression of four top candidate genes, C1orf130, $C N T F, C O L 4 A 3$, and COL4A4, was verified by quantitative reverse transcription PCR.

Conclusion: NF2 mutations are not directly involved in the cystic formation of vestibular schwannoma. In addition, the differential gene expression of cystic vestibular schwannoma reported in our study may provide useful insights into the molecular mechanism underlying this process.

Genet Med advance online publication 12 September 2013

Key Words: cystic formation; differential gene expression; NF2 mutation; vestibular schwannoma

\section{INTRODUCTION}

Vestibular schwannoma (VS), one of the most common intracranial tumors in the lateral skull base, ${ }^{1}$ has a steadily increasing incidence of up to 22.8 tumors per million people annually due to the innovation of diagnostic methods during the past several decades. ${ }^{2,3}$ The tumor arises from the vestibular branch of the eighth cranial nerve. Asymmetric hearing loss, tinnitus, disequilibrium, facial numbness, and weakness are presenting symptoms. Furthermore, hydrocephalus, brain stem compression, herniation, and death are potential hazards if the tumor is large enough. On the basis of the clinical features, such as tumor size, tumor growth rate, hearing level, and the patient's individual life situation and preferences, the management options of VS could be chosen from "wait and scan," stereotactic radiosurgery, fractioned radiotherapy, and surgical resection.,

Depending on neuroradiological appearance, VS could be categorized as one of two subtypes: solid VS (SVS) and cystic VS (CVS). The more formidable subtype, CVS, accounts for 6.8 to $20.4 \%$ of VS based on several reports with larger series ${ }^{6-9}$ and is notorious for aggressive clinical features, including rapid tumor growth $(2-6 \mathrm{~mm} /$ year $),{ }^{10}$ much more severe symptoms, ${ }^{11,12}$ intensive adherence to the facial nerve, ${ }^{13}$ and difficulty predicting its biological behavior. It often presents a dilemma for neurotologists to choose optimal management: the "wait and scan" policy may delay the optimal therapeutic time due to sudden or persistent fast growth. Stereotactic radiosurgery or fractioned radiotherapy is not suitable for CVS because it may accelerate the expansion of the tumor. ${ }^{13}$ Surgical resection rarely gets satisfactory postoperative functional outcomes in CVS..$^{6-8,11,14,15}$

The NF2 gene encodes a 595 -amino-acid protein known as schwannomin or merlin, which is similar to a class of 4.1 superfamily of protein (moesin, ezrin, and radixin). ${ }^{16}$ It is implicated in the cell and cell-cycle control processes such as adhesion, migration, cell-cell contact, spreading, proliferation, and signal transduction. ${ }^{17}$ Mutations in NF2 gene play a critical role in VS pathogenesis ${ }^{18-20}$ and were the cause of VS in $\sim 66 \%$ of cases. ${ }^{21}$ They can occur through inheritance from an affected parent, mosaic or de novo zygote mutation, mutations during embryogenesis, or somatic mutations throughout life after birth. ${ }^{22,23}$

Despite extensive studies of NF2 mutations in VS, the role of NF2 or any other genes in cystic formation of VS remains elusive. In our previous clinical studies, ${ }^{15}$ we had retrospectively analyzed the aggressive clinical features and unfavorable surgical outcomes of large or giant CVS. In this study, we intended to evaluate the role of NF2 mutations in the pathogenesis of CVS by comparison of the mutation spectrum of NF2 between CVS and SVS. Differential gene expression of CVS versus SVS was investigated

${ }^{1}$ Department of Otolaryngology-Head \& Neck Surgery, Xinhua Hospital, Shanghai Jiaotong University School of Medicine, Shanghai, China; ${ }^{2}$ Ear Institute, Shanghai Jiaotong University School of Medicine, Shanghai, China. Correspondence: Hao Wu (wuhao622@sina.cn) or Tao Yang (yangtfxl@sina.com) 
using the cDNA microarray analysis. Top candidate genes were verified by quantitative reverse transcription PCR (qRT-PCR).

\section{MATERIALS AND METHODS}

\section{Patients}

We retrospectively reviewed 145 patients with sporadic VS, including 31 patients with CVS and 114 with SVS, who underwent surgical resection in our department between June 2008 and June 2011. Magnetic resonance imaging was routinely performed to make the neuroimaging diagnosis (Figure 1). A tumor was labeled as CVS or SVS depending on two key points: ${ }^{8}$ (i) presence or absence of hypodense/hypointense areas on T1-weighted postgadolinium magnetic resonance imaging and (ii) identification of cystic or solid elements during the surgery. Enhancement of the cyst wall was used as the imaging marker to differentiate CVS from arachnoid cysts and epidermoids. ${ }^{24}$

Tumor size measurement was based on the largest diameter of the extrameatal portion in cerebellopontine angle from T1-weighted magnetic resonance imaging with gadolinium. Facial nerve function was assessed from grade I (normal) to VI (worst) using the House-Brackmann grading system. $\cdot{ }^{25}$ Hearing level was evaluated from class A (normal) to D (worst) using the American Academy of Otolaryngology-Head and Neck surgery classification. ${ }^{26}$

All tumor tissue samples underwent routine histology, and the diagnoses were confirmed by a pathologist. All patients signed an informed consent form releasing tumor tissue and blood sample for research purposes. This study was approved by the ethics committee of the Shanghai Jiaotong University School of Medicine, Xinhua Hospital, Shanghai, China.

\section{Mutational screening of the NF2 gene}

DNA samples were extracted from both fresh-frozen tumor tissues and blood samples of all patients using the the TIANamp genomic DNA kit (Tiangen Biotech, Beijing, China). All 17 exons of NF2 were PCR amplified and subjected to bidirectional sequencing as previously described. ${ }^{27}$ Sequence data were analyzed using Sequencer 4.9 software (Genecode, Ann Arbor, MI).

\section{cDNA microarray analysis}

cDNA microarray analysis was performed in 11 CVSs (T114, T115, T145, T116, T134, T127, T137, T141, T132, T140, and T142) and 6 SVSs (T125, T126, T128, T135, T144, and T139). Tumor tissues were harvested from the subcapsular part and immediately immersed in the RNAlater RNA stabilization reagent (Qiagen, Shanghai, China) and stored at $4{ }^{\circ} \mathrm{C}$ until use. mRNA samples were extracted using the TRIzol reagent (Invitrogen, Carlsbad, CA) and reverse transcribed to complementary DNA using the Quantscript RT kit (Tiangen Biotech). Hybridization was performed using the Illumina Human-12T v4 Expression BeadChip system (Illumina, San Diego, CA), which contains 47,231 probes per array product. Normalization algorithms were used to transform sample signals to minimize the effects of variation arising from nonbiological factors. Analysis of gene expression profiles was completed by the Illumina BeadStudio module application (Illumina). A twofold or greater difference in the intensity of gene expression between the CVS and SVS was sought. Overexpressed or underexpressed genes were cataloged. The $t$-test with unpaired unequal variance (Welch) was used to calculate $P$ values. Adjusted for multiple testing, the inclusion criteria of the differentially expressed

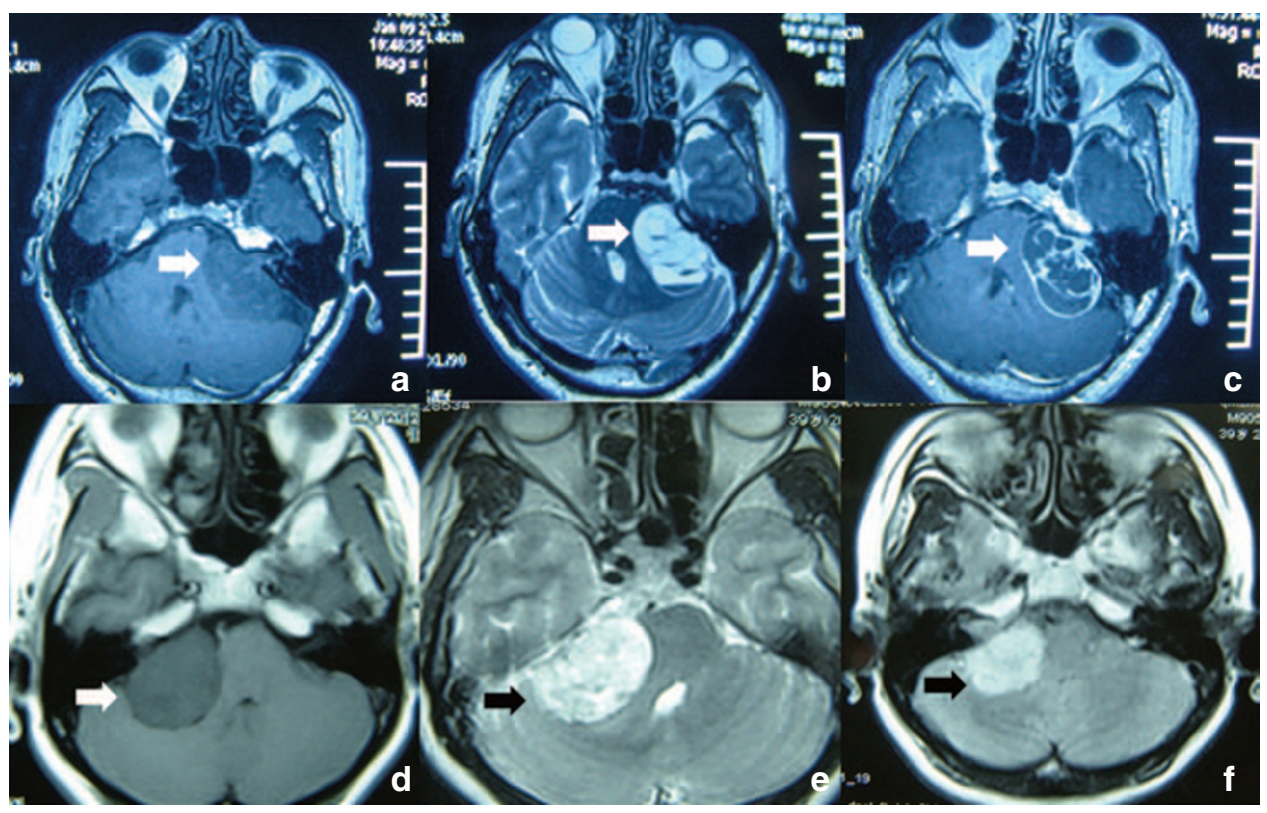

Figure 1 Magnetic resonance imaging of representative cystic and solid vestibular schwannomas. (a-c) A left cystic vestibular schwannoma (CVS) $45 \mathrm{~mm}$ in diameter. It manifested a (a) heterogeneous hypodensity lesion in axial T1-weighted image and (b) multicystic hyperdensity lesion in axial T2-weighted image. (c) The enhancement of the cyst wall and multicystic tumors is shown in axial T1-weighted postgadolinium. (d-f) A right solid vestibular schwannoma (SVS) $40 \mathrm{~mm}$ in diameter. It manifested a (d) homogeneous hypodensity lesion in axial T1-weighted image, (e) hyperdensity lesion in axial T2-weighted image, and (f) homogeneous enhanced lesion in axial T1-weighted postgadolinium. 
genes were $P<0.005$ and fold change $>2$, or $P<0.01$ and fold change $>10$. Genes with the largest fold changes and/or those known to be involved in cell growth, proliferation, and oncogenesis signaling pathways were selected and further evaluated by qRT-PCR.

\section{qRT-PCR}

The differential expression of five top candidate genes, CLORF130, CNTF, FGFBP2, COL4A3, and COL4A4, was further evaluated by SYBR green-based qRT-PCR. mRNA samples were extracted and reverse transcribed as described in the cDNA analysis. qRT-PCR primers and probes of each gene were designed using ABI Primer Express Software v2.0 (Applied Biosystems, Foster City, CA; sequences shown in Supplementary Table S1 online). qRT-PCR analysis of the cDNA samples was performed using the ABI Prism 7700 sequence detection system (Applied Biosystems) following standard procedure..$^{28}$ Data were analyzed using the ABI Prism sequence detection system software (Applied Biosystems). Quantitative expression data of each specific target were obtained for each cDNA sample. Expression of the $\beta$-actin gene was used as endogenous normalization control.
Mann-Whitney test was used to compare the median expression values. The comparative threshold of cycle $\left(C_{\mathrm{T}}\right)$ method was used to determine any difference in target expression between CVS and SVS. All values were $\log _{2}$ transformed before statistical analysis.

\section{Statistical analysis}

Statistical analysis of the study was performed in the facilities of the Molecular Biology of Hearing and Deafness Research, Xinhua Hospital. Fisher's exact test and odds ratio calculation were used to compare surgical outcomes and NF2 mutation rate. $P$ values were presented as the result of the two-tailed analysis. Mann-Whitney test was used to compare the median expression values in qRT-PCR. Pearson correlation was used to compare the correlation between results of cDNA microarray and qRT-PCR.

\section{RESULTS}

CVS was associated with more severe symptoms than SVS As shown in Table 1, patients with CVS had shorter duration of symptoms (mean: 1.4 years; 95\% confidence interval: $0.1-8$ years; $P=0.020$ ), worse hearing level (class $\mathrm{D}$ in

Table 1 Clinical data of patients with sporadic vestibular schwannoma

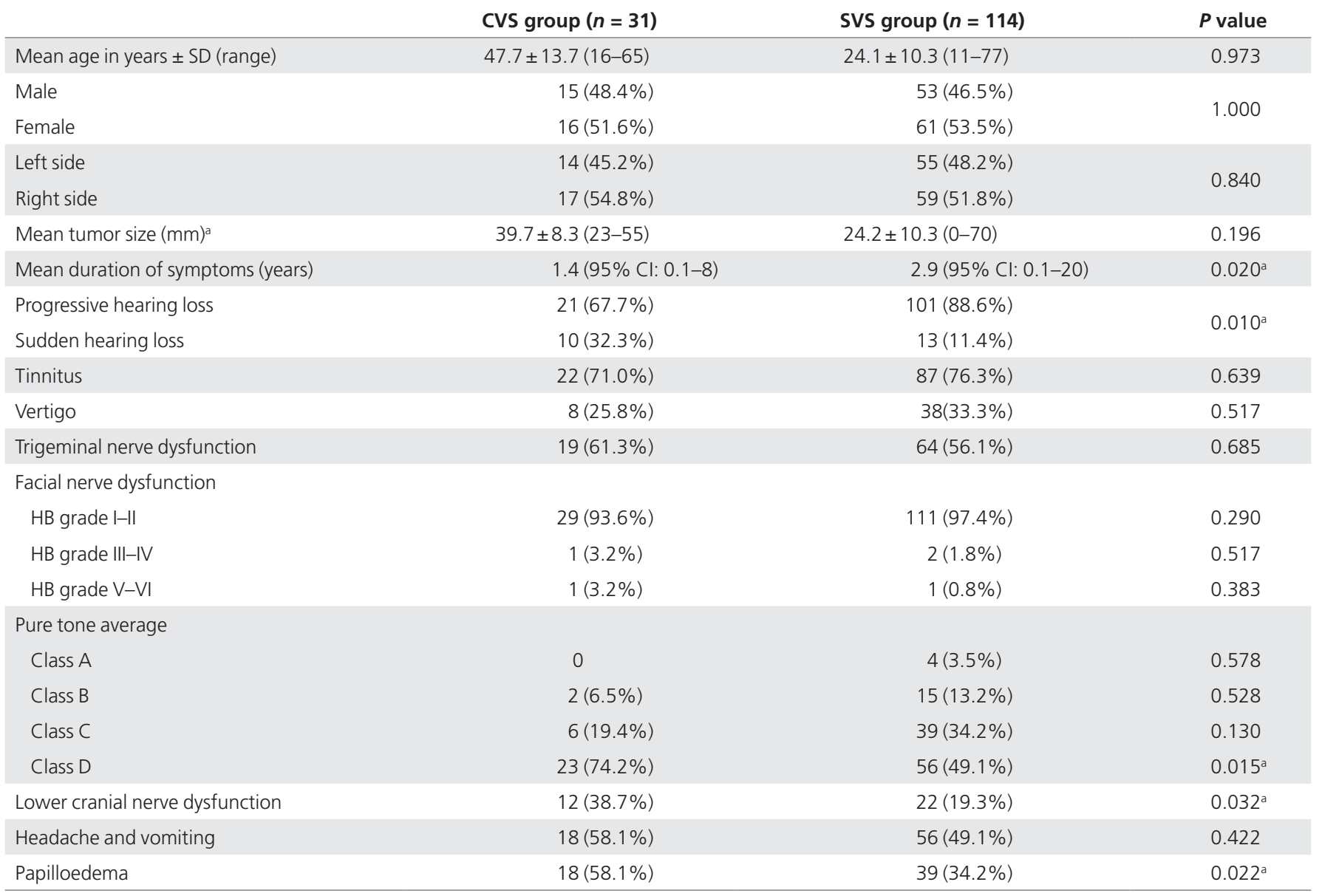

$\mathrm{Cl}$, confidence interval; CVS, cystic vestibular schwannoma; HB, House-Brackmann facial nerve grading system; SVS, solid vestibular schwannoma. a $P<0.05$ 
74.2\%; $P=0.015)$, higher occurrence of sudden hearing loss $(32.3 \% ; P=0.010)$, lower cranial nerve dysfunction $(38.7 \% ; P=$ $0.032)$, and papilloedema (58.1\%; $P=0.022)$.

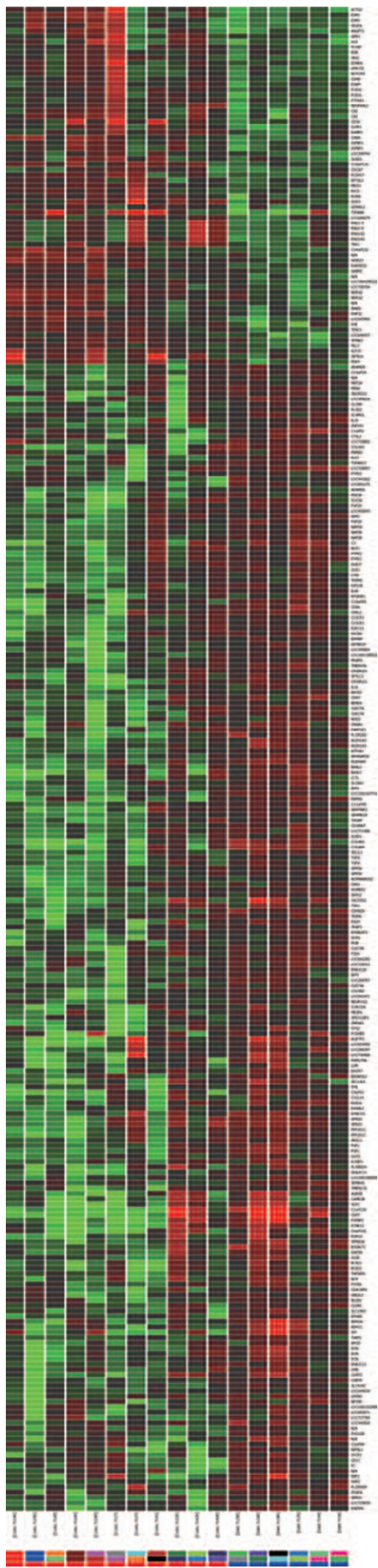

NF2 mutations were not directly involved in cystic formation of VS

In our mutation screening of NF2 in 145 patients with sporadic VSs (31 CVSs and 114 SVSs), only somatic mutations were detected from the tumor DNA samples. No constitutive mutation of NF2 was detected from the blood DNA samples. Single heterozygous mutations of NF2 were detected in 9 CVSs and 38 SVSs, whereas compound heterozygous mutations were detected in 1 SVS, and apparent homozygous mutations were detected in 2 CVSs (Supplementary Table S2 online). The mutation detection rate of NF2 was $34.5 \%$ (50/145) in overall VSs, $35.5 \%$ (11/31) in CVSs, and 34.2\% (39/114) in SVSs. There was no significant difference of the mutation rates between CVSs and SVSs (Fisher's exact test; $P=1.000$ ). Although most NF2 mutations identified were truncating mutations (nonsense, frameshift, and splicing-site mutations), we did not observe any significant difference in the percentages of truncating and nontruncating mutations between those two groups. These results suggested that NF2 mutations are not directly involved in the cystic formation of VS.

\section{Differential gene expression between CVS and SVS}

A total of 17 representative tumors (11 CVSs and 6 SVSs; see clinical data in Supplementary Table S3 online) were subjected to differential gene expression analysis using the cDNA microarray method. A hierarchical cluster of significantly up- or downregulated probes/genes in CVSs is presented in Figure 2. A total of 34,690 genes were assessed per array. Of the 46 differentially expressed genes in CVSs (Supplementary Table S4 online), 35 (76.1\%) were downregulated and 11 (23.9\%) were upregulated. All differentially expressed genes were matched against the Gene Ontology Consortium database ${ }^{29}$ using the built-in annotation functionality. Gene annotation enrichment analysis of the clustered genes revealed significant enrichment of annotation for five genes in pathways of cancer $(P=$ 0.0000902): one in cell adhesion molecules $(P=0.0021)$, three in cytokine-cytokine receptor interaction $(P=0.0056)$, and one in extracellular matrix-receptor interaction $(P=0.0326)$.

\section{qRT-PCR experiment confirmed four differentially expressed genes between CVS and SVS}

The top five candidate genes with the highest differential expression ( $>10$-fold changes and $P \leq 0.001)$ were chosen for further verification by qRT-PCR. All five genes-the chromosome 1 open reading frame 130 gene CLORF130, the ciliary neurotrophic factor gene CNTF, the fibroblast growth factor binding protein 2 gene FGFBP2, the collagen type IV a 3 gene

Figure 2 Heat map of the clustered genes. Of the 46 differentially expressed genes in vestibular schwannoma tumor tissue, 35 were downregulated and 11 upregulated. The color intensities of the heat map indicate the degree of up- (red) or downregulation (green) in CVSs. Each column of colored squares represents one tissue sample. Seventeen samples from left to right are as follows: 11 CVSs (T114, T115, T145, T116, T134, T127, T137, T141, T132, T140, and T142), 6 SVSs (T125, T126, T128, T135, T144, and T139). CVS, cystic vestibular schwannoma; SVS, solid vestibular schwannoma 
Table 2 Top five differentially expressed genes in cystic vestibular schwannoma by microarray analysis

\begin{tabular}{lccccl} 
Probe ID & Gene symbol & Cytoband & Fold change & $\boldsymbol{P}$ value & Function \\
\hline ILMN_3246014 & Clorf130 & 2p13.1b & -22.30 & 0.001 & Unknown function \\
\hline ILMN_1773113 & CNTF & $8 p 21.2 \mathrm{~d}$ & -16.58 & 0.004 & Cytokine-cytokine receptor interaction \\
\hline ILMN_1761945 & FGFBP2 & $8 \mathrm{p} 21.2 \mathrm{~d}$ & -13.40 & 0.007 & Cytotoxic lymphocyte-mediated immunity \\
\hline ILMN_1662731 & COL4A3 & $4 \mathrm{q} 23 \mathrm{~b}$ & -12.92 & 0.003 & Pathways in cancer \\
\hline ILMN_1778308 & COL4A4 & $1 \mathrm{q} 23.1 \mathrm{~g}-\mathrm{q} 23.2 \mathrm{a}$ & -10.43 & 0.001 & Pathways in cancer \\
\hline
\end{tabular}

COL4A3, and the collagen type IVa 4 gene COL4A4-were downregulated in CVS and participate in pathways of oncogenesis or cytokine-cytokine receptor interaction (Table 2). Our qRT-PCR analysis confirmed significant downregulation in four candidate genes, CLORF130 (fold change $=-2.81 ; P=$ $\left.9.5 \times 10^{-10}\right), C N T F\left(\right.$ fold change $\left.=-2.77 ; P=5.7 \times 10^{-5}\right), C O L 4 A 3$ (fold change $=-4.10 ; P=0.017$ ), and COL4A4 (fold change $=$ $-1.56 ; P=0.003$ ), whereas the remaining FGFBP2 gene showed borderline significance (fold change $=-1.75 ; P=0.062$ ) of differential expression (Figure 3). For three genes, C1orf130 $\left(R^{2}=\right.$ $0.572 ; P=0.007), \operatorname{CNTF}\left(R^{2}=0.529 ; P=0.011\right)$, and COL $4 A 4$ $\left(R^{2}=0.594 ; P=0.005\right)$, the expression levels had strong correlation between those measured by the cDNA microarray and the qRT-PCR (Figure 3).

\section{DISCUSSION}

Sporadic VS is an intracranial benign tumor that originates from Scapa ganglion of vestibular nerve in internal acoustic meatus. During its growth process, the tumor could oppress and adhere to adjacent tissues such as trigeminal nerve, facial nerve, vestibulo-cochlear nerve, lower cranial nerves, and brain stem, which evoke associated central nerve dysfunction, hydrocephalus, and even encephalocele. As a crucial feature for surgery, the CVS usually means aggressive clinical features and worse postoperative outcomes..$^{10-13}$ The molecular mechanism underlying the cystic degeneration of VS, however, remains unclear.

Since NF2 mutation is the major cause of VS, we compared the mutation screening results of NF2 between CVSs and SVSs. Because we did not evaluate the loss of heterozygosity of NF2, the percentage of NF2 mutations identified in our study was relatively low as compared with some other reports. ${ }^{30}$ Nevertheless, our study showed that although $34.5 \%$ of sporadic VSs had at least one NF2 mutation, no significant difference was observed between these two groups (35.5 vs. $34.2 \%$; $P$ $=1.000$ ). Furthermore, the proportion of mutation types (truncating and nontruncating mutations) was also similar between them. Our finding suggested that although NF2 mutation is central for oncogenesis of VS, it may not directly participate in the cystic degeneration of VS.

To detect key genes involved in cystic degeneration in VS, we obtained a list of differentially expressed genes of CVS by cDNA microarray analysis. In contrast to a previous study in which no gene was reported differentially expressed between 7 CVSs and 18 noncystic VSs ${ }^{31}$ we identified 46 differentially expressed genes in CVSs (see Supplementary Table S4 online). qRT-PCR analysis subsequently verified the differential expression in the top five candidate genes including C1orf130, CNTF, FGFBP2, COL4A3, and COL4A4.

The C1orf130 gene showed the highest downregulation (fold change $=-2.81 ; P=9.5 \times 10^{-10}$, based on qRT-PCR results) in CVS. It encodes noncompact myelin-associated protein, which had another alias, myelin glycoprotein. As a transmembrane protein and a novel myelination-associated transcript, the myelin glycoprotein is expressed in Schwann cells and is a component of peripheral myelin. ${ }^{32}$ Its expression is regulated by early growth response 2 during development and after nerve injury but appears to be negligible in the brain. ${ }^{32}$ Both overexpression and knockdown of myelin glycoprotein could result in significantly less myelin formation, which may be involved in the biological process of cystic degeneration of VS.

The CNTF gene also manifested a high degree of downregulation (fold change $=-2.77 ; P=5.7 \times 10^{-5}$ ) in CVS. It encodes the CNTF protein, which is a polypeptide hormone whose actions appear to be restricted to the nervous system, where it promotes neurotransmitter synthesis and neurite outgrowth in certain neuronal populations. The CNTF protein is a potent survival factor for neurons and oligodendrocytes and may be involved in reducing tissue destruction during inflammatory attacks and in affecting growth and differentiation of various neoplasms. ${ }^{33}$ Dozio et al. ${ }^{34}$ reported that it presents a similar three-dimensional fold structure as class I cytokines, which participate in the complex control of the reproductive function by affecting the development and function of the hypothalamus-pituitary system at different ontogenic times and anatomical sites.

The COL4A3 and COL4A4 genes encode two of the six subunits of type IV collagen, the major structural component of basement membranes. Type IV collagen has been linked not only to specific kidney pathologies, such as the autosomal-recessive form of Alport syndrome (hereditary glomerulonephropathy) and familial benign hematuria (thin basement membrane disease), but also to tumor invasion and metastasis, including gastric, colorectal, ovarian, and breast cancers, as well as welldifferentiated lung carcinomas. ${ }^{35,36}$ It appears that type IV collagen could control reproductive function at different ontogenic times and anatomical sites and plays functional roles in cell adhesion, cell differentiation, and tissue development. ${ }^{37,38}$ In our study, we observed a significant downregulation of COL4A3 (fold change $=-4.10, P=0.017$ ) and COL4A4 (fold change $=$ $-1.56, P=0.003)$ in CVSs, which also suggested that those two genes may be involved in the cystic formation of VS.

The FGFBP2 gene showed borderline significance (fold change $=-1.75 ; P=0.062)$ of downregulation in CVS. Its protein 
a
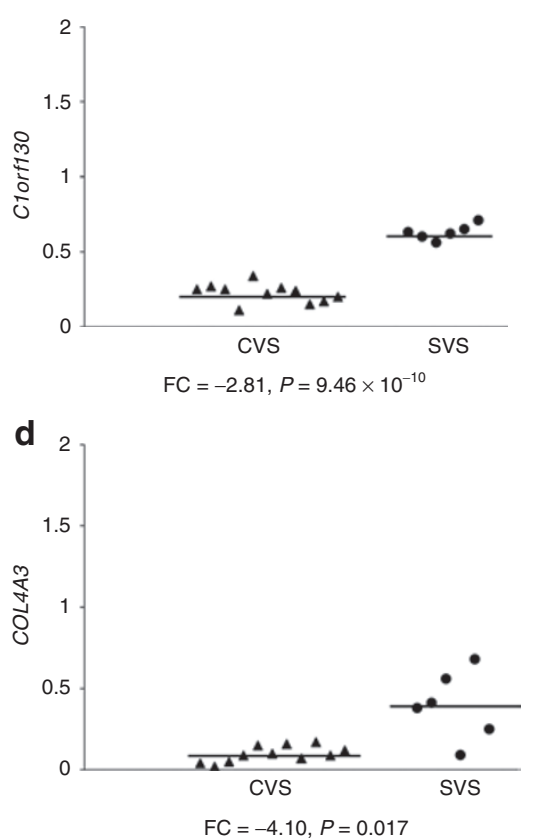

g

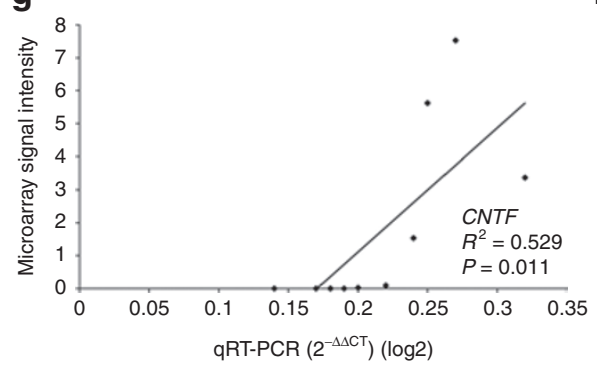

b

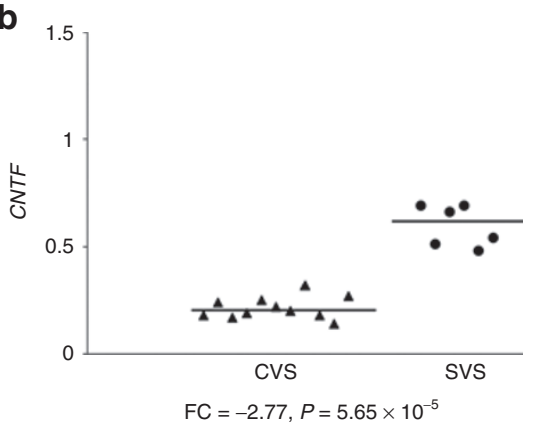

e

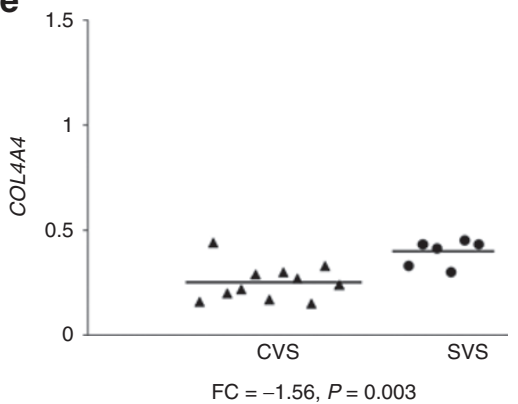

h

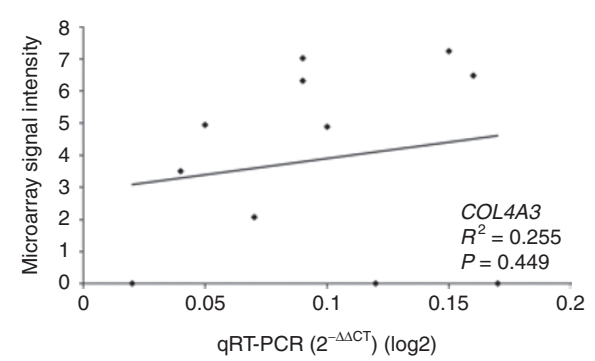

C

f

i
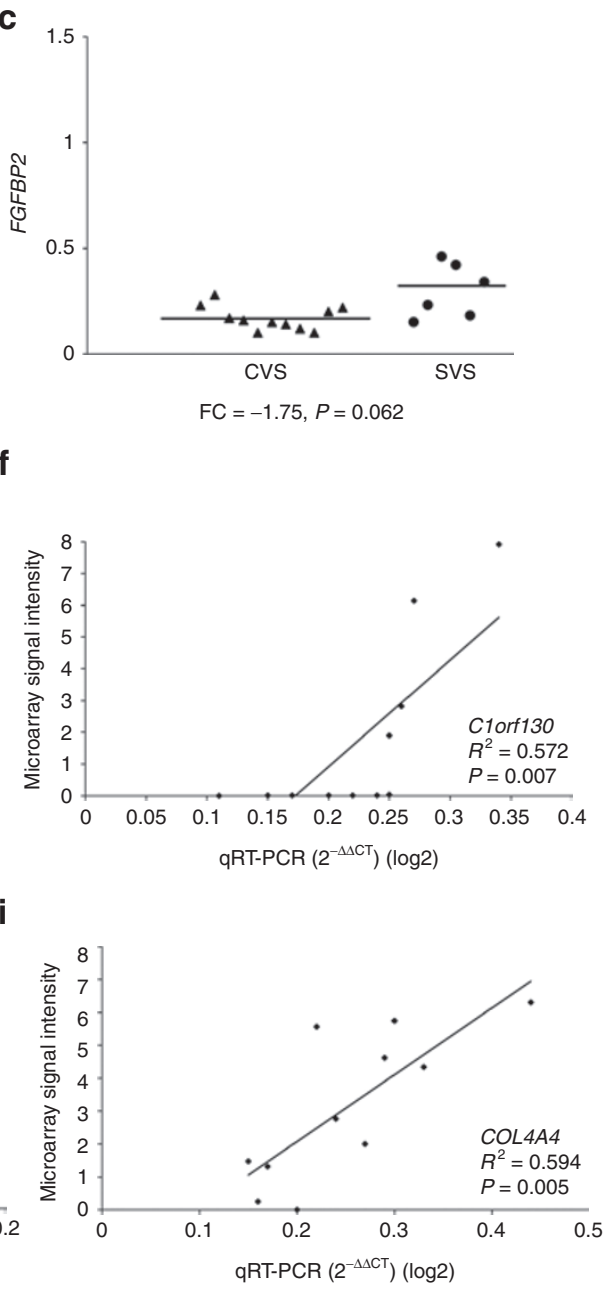

Figure 3 qRT-PCR analysis of candidate genes. (a-e) qRT-PCR verification of the top 5 differentially expressed candidate genes in 11 cystic vestibular schwannomas (CVSs), 6 solid vestibular schwannomas (SVSs), and 3 normal peripheral nerves (NNs). The expression level of each gene ( $y$-axis) was normalized to that of the $\beta$-actin control, with the median expression in NN set as 1. P values (bottom) were calculated by Mann-Whitney test to compare the expression level between CVSs and SVSS. FC, fold change. ( $\mathbf{f}-\mathbf{i})$ The correlation between the expression levels of the candidate genes measured by qRT-PCR and the cDNA microarray. All values were $\log _{2}$ converted before the statistical analysis. qRT-PCR, quantitative reverse transcription PCR.

is expressed in cytotoxic $\mathrm{T}$ lymphocytes and natural killer cells and may be involved in cytotoxic lymphocyte-mediated immunity. Several studies found that high expression of this protein was positively correlated with survival in high-grade gliomas and histological type, clinical stage, and good prognosis in ovarian carcinomas. ${ }^{39,40}$

\section{Conclusions}

Our study showed that NF2 mutations may not be directly involved in the cystic formation of VS. In addition, a large number of genes with differential gene expression between CVSs and SVSs, including Clorf130, CNTF, COL4A3, and COL4A4, are potential candidates for this biological process and may warrant further investigation.

\section{SUPPLEMENTARY MATERIAL}

Supplementary material is linked to the online version of the paper at http://www.nature.com/gim

\section{ACKNOWLEDGMENTS}

This research was supported by a grant from the National Basic Research Program of China (2011CB504501 to H.W.), grants from the National Science Foundation of China (81170924 and 30973307 to H.W., 81222010 to T.Y.), grants from the Shanghai Municipal Science and Technology Commission (11PJ1407000 to T.Y.), and the Oriental Scholar Project from the Shanghai Municipal Education Commission (T.Y.).

\section{DISCLOSURE}

The authors declare no conflict of interest.

\section{REFERENCES}

1. Mahaley MS Jr, Mettlin C, Natarajan N, Laws ER Jr, Peace BB. Analysis of patterns of care of brain tumor patients in the United States: a study of the Brain Tumor Section of the AANS and the CNS and the Commission on Cancer of the ACS. Clin Neurosurg 1990;36:347-352.

2. Evans DG, Moran A, King A, Saeed S, Gurusinghe N, Ramsden R. Incidence of vestibular schwannoma and neurofibromatosis 2 in the North West of England 
over a 10-year period: higher incidence than previously thought. Otol Neurotol 2005;26:93-97.

3. Stangerup SE, Tos M, Thomsen J, Caye-Thomasen P. True incidence of vestibular schwannoma? Neurosurgery 2010;67:1335-1340.

4. van de Langenberg R, de Bondt BJ, Nelemans PJ, Dohmen AJ, Baumert BG, Stokroos RJ. Predictors of volumetric growth and auditory deterioration in vestibular schwannomas followed in a wait and scan policy. Otol Neurotol 2011;32(2):338-344.

5. Müller S, Arnolds J, van Oosterhout A. Decision-making of vestibular schwannoma patients. Acta Neurochir (Wien) 2010;152:973-984.

6. Sinha S, Sharma BS. Cystic acoustic neuromas: surgical outcome in a series of 58 patients. J Clin Neurosci 2008;15:511-515.

7. Jones SE, Baguley DM, Moffat DA. Are facial nerve outcomes worse following surgery for cystic vestibular schwannoma? Skull Base 2007;17:281-284.

8. Piccirillo $E$, Wiet MR, Flanagan $S$, et al. Cystic vestibular schwannoma: classification, management, and facial nerve outcomes. Otol Neurotol 2009;30:826-834.

9. Jian BJ, Sughrue ME, Kaur R, et al. Implications of cystic features in vestibular schwannomas of patients undergoing microsurgical resection. Neurosurgery 2011;68:874-80; discussion 879.

10. Selesnick SH, Johnson G. Radiologic surveillance of acoustic neuromas. Am J Otol 1998;19:846-849.

11. Moon KS, Jung S, Seo SK, et al. Cystic vestibular schwannomas: a possible role of matrix metalloproteinase-2 in cyst development and unfavorable surgical outcome. J Neurosurg 2007;106:866-871.

12. Mehrotra N, Behari S, Pal L, Banerji D, Sahu RN, Jain VK. Giant vestibular schwannomas: focusing on the differences between the solid and the cystic variants. Br J Neurosurg 2008;22:550-556.

13. de Ipolyi AR, Yang I, Buckley A, Barbaro NM, Cheung SW, Parsa AT. Fluctuating response of a cystic vestibular schwannoma to radiosurgery: case report. Neurosurgery 2008;62:E1164-5; discussion E1165.

14. Fundová P, Charabi S, Tos M, Thomsen J. Cystic vestibular schwannoma: surgical outcome. J Laryngol Otol 2000;114:935-939.

15. Zhang Z, Wang Z, Huang Q, Yang J, Wu H. Removal of large or giant sporadic vestibular schwannomas via translabyrinthine approach: a report of 115 cases. ORL J Otorhinolaryngol Relat Spec 2012;74:271-277.

16. Bretscher A, Edwards K, Fehon RG. ERM proteins and merlin: integrators at the cell cortex. Nat Rev Mol Cell Biol 2002;3:586-599.

17. McClatchey Al, Giovannini M. Membrane organization and tumorigenesis-the NF2 tumor suppressor, Merlin. Genes Dev 2005;19:2265-2277.

18. Trofatter JA, MacCollin MM, Rutter JL, et al. A novel moesin-, ezrin-, radixinlike gene is a candidate for the neurofibromatosis 2 tumor suppressor. Cell 1993:72:791-800.

19. Jacoby LB, MacCollin M, Louis DN, et al. Exon scanning for mutation of the NF2 gene in schwannomas. Hum Mol Genet 1994;3:413-419.

20. Cayé-Thomasen P, Borup R, Stangerup SE, Thomsen J, Nielsen FC. Deregulated genes in sporadic vestibular schwannomas. Otol Neurotol 2010;31:256-266.

21. Gutmann DH, Giordano MJ, Fishback AS, Guha A. Loss of merlin expression in sporadic meningiomas, ependymomas and schwannomas. Neurology 1997:49:267-270.

22. Evans DG, Bowers N, Huson SM, Wallace A. Mutation type and position varies between mosaic and inherited NF2 and correlates with disease severity. Clin Genet 2013:83:594-595.
23. Evans DG, Maher ER, Baser ME. Age related shift in the mutation spectra of germline and somatic NF2 mutations: hypothetical role of DNA repair mechanisms. J Med Genet 2005:42:630-632.

24. Falcioni A, Piccirillo E, Mancini F. Cystic vestibular schwannoma. Am J Otol 2000;21:595-596.

25. House JW, Brackmann DE. Facial nerve grading system. Otolaryngol Head Neck Surg 1985;93:146-147.

26. American Academy of Otolaryngology-Head and Neck Surgery Foundation. Committee on Hearing and Equilibrium guidelines for the evaluation of hearing preservation in acoustic neuroma (vestibular schwannoma). Otolaryngol Head Neck Surg 1995;113(3):179-180.

27. Sestini R, Provenzano A, Bacci C, Orlando C, Genuardi M, Papi L. NF2 mutation screening by denaturing high-performance liquid chromatography and highresolution melting analysis. Genet Test 2008;12:311-318.

28. Patel AK, Alexander TH, Andalibi A, Ryan AF, Doherty JK. Vestibular schwannoma quantitative polymerase chain reaction expression of estrogen and progesterone receptors. Laryngoscope 2008;118:1458-1463.

29. Ashburner M, Ball CA, Blake JA, et al. Gene ontology: tool for the unification of biology. The Gene Ontology Consortium. Nat Genet 2000;25:25-29.

30. Hadfield KD, Smith MJ, Urquhart JE, et al. Rates of loss of heterozygosity and mitotic recombination in NF2 schwannomas, sporadic vestibular schwannomas and schwannomatosis schwannomas. Oncogene 2010;29:6216-6221.

31. Aarhus M, Bruland O, Sætran HA, Mork SJ, Lund-Johansen M, Knappskog PM. Global gene expression profiling and tissue microarray reveal novel candidate genes and down-regulation of the tumor suppressor gene CAV1 in sporadic vestibular schwannomas. Neurosurgery 2010;67:998-1019.

32. Ryu EJ, Yang M, Gustin JA, et al. Analysis of peripheral nerve expression profiles identifies a novel myelin glycoprotein, MP11. J Neurosci 2008;28:7563-7573.

33. Weis J, Schönrock LM, Züchner SL, et al. CNTF and its receptor subunits in human gliomas. J Neurooncol 1999;44:243-253.

34. Dozio E, Ruscica M, Galliera E, Corsi MM, Magni P. Leptin, ciliary neurotrophic factor, leukemia inhibitory factor and interleukin-6: class-I cytokines involved in the neuroendocrine regulation of the reproductive function. Curr Protein Pept Sci 2009; 10:577-584.

35. Parkin JD, San Antonio JD, Pedchenko V, Hudson B, Jensen ST, Savige J. Mapping structural landmarks, ligand binding sites, and missense mutations to the collagen IV heterotrimers predicts major functional domains, novel interactions, and variation in phenotypes in inherited diseases affecting basement membranes. Hum Mutat 2011:32:127-143.

36. Nie XC, Wang JP, Zhu W, et al. COL4A3 expression correlates with pathogenesis, pathologic behaviors, and prognosis of gastric carcinomas. Hum Pathol 2013:44:77-86.

37. Gioia M, Monaco S, Van Den Steen PE, et al. The collagen binding domain of gelatinase A modulates degradation of collagen IV by gelatinase B. J Mol Biol 2009;386:419-434.

38. Prockop DJ, Kivirikko KI. Collagens: molecular biology, diseases, and potentials for therapy. Annu Rev Biochem 1995;64:403-434.

39. Yamanaka R, Arao T, Yajima N, et al. Identification of expressed genes characterizing long-term survival in malignant glioma patients. Oncogene 2006;25:5994-6002.

40. Elgaaen BV, Haug KB, Wang J, et al. POLD2 and KSP37 (FGFBP2) correlate strongly with histology, stage and outcome in ovarian carcinomas. PLOS ONE 2010;5:e13837. 